TITLE:

\title{
Laser-Induced Kondo Effect in Ultracold Alkaline-Earth Fermions.
}

$\operatorname{AUTHOR}(S):$

Nakagawa, Masaya; Kawakami, Norio

\section{CITATION:}

Nakagawa, Masaya ...[et al]. Laser-Induced Kondo Effect in Ultracold Alkaline-Earth Fermions.. Physical review letters 2015, 115(16): 165303.

ISSUE DATE:

2015-10-16

URL:

http://hdl.handle.net/2433/203043

RIGHT:

C 2015 American Physical Society 


\title{
Laser-Induced Kondo Effect in Ultracold Alkaline-Earth Fermions
}

\author{
Masaya Nakagawa and Norio Kawakami \\ Department of Physics, Kyoto University, Kyoto 606-8502, Japan
}

(Received 10 June 2015; published 16 October 2015)

\begin{abstract}
We demonstrate that laser excitations can coherently induce a novel Kondo effect in ultracold atoms in optical lattices. Using a model of alkaline-earth fermions with two orbitals, it is shown that the optically coupled two internal states are dynamically entangled to form the Kondo-singlet state, overcoming the heating effect due to the irradiation. Furthermore, a lack of $\mathrm{SU}(N)$ symmetry in the optical coupling provides a peculiar feature in the Kondo effect, which results in spin-selective renormalization of effective masses. We also discuss the effects of interorbital exchange interactions, and reveal that they induce novel crossover or reentrant behavior of the Kondo effect owing to control of the coupling anisotropy. The laserinduced Kondo effect is highly controllable by tuning the laser strength and the frequency, and thus offers a versatile platform to study the Kondo physics using ultracold atoms.
\end{abstract}

DOI: 10.1103/PhysRevLett.115.165303

PACS numbers: 67.85.-d, 37.10.Jk, 75.30.Mb

Even after a long history, the Kondo effect is still one of the central problems in condensed matter physics since it captures many essential aspects of strongly correlated systems. This effect arises from the screening of a localized spin by a surrounding fermion cloud due to antiferromagnetic coupling, forming an emergent entangled state called the Kondo singlet [1]. In some rare-earth compounds where localized spins are periodically aligned, the Kondo effect takes place at every lattice site, and the quasiparticle band arising from the Kondo singlets forms a strongly renormalized heavy-fermion liquid which gives rise to diverse phenomena such as quantum criticality [2]. On the other hand, recent development of manipulation techniques using intense laser fields offers versatile methods to engineer intriguing states of quantum matter [3,4]. Laser-dressed states are a useful resource both in electronic and coldatomic systems [5-10], but controlling the nature of these states in strongly correlated systems is challenging since we must deal with both strong-correlation effects and the influence of driving nonperturbatively [11]. The Kondo effect under irradiation provides a prototypical example of this problem. For example, in systems such as quantum dots, its nonequilibrium properties [12-15] and optical phenomena in the Kondo effects [16-19] were investigated.

In this Letter, we propose a novel method to engineer the Kondo effect using intense laser fields and ultracold fermions in optical lattices. Our main idea is to realize the (effective) antiferromagnetic coupling between the localized spins and the fermion cloud using optical transitions induced by the laser. We show that the optical coupling dynamically entangles the spins with the cloud due to the Kondo effect, and thereby realizes a heavyfermion liquid. A possible drawback in this scheme is the heating effect caused by the application of the laser. Here we find that the dynamically induced heavy-fermion liquid indeed persists under the irradiation, even if there is a substantial heating effect. Since the Kondo coupling can be tuned by the laser strength and frequency, the laser-induced Kondo effect offers a highly controllable scheme to generate the Kondo states in ultracold atoms. Moreover, it gives a natural platform to study real-time dynamics of the Kondo effect in various nonequilibrium situations.

As a suitable system to realize the Kondo effect using light-matter interactions, we focus on ultracold alkalineearth-like atoms (AEAs) [20-28] in optical lattices. AEAs have an electronic ground state $\left({ }^{1} S_{0}\right)$ and a long-lived metastable excited state $\left({ }^{3} P_{0}\right)$. The stable and metastable states compose a two-orbital system, and the coherent optical process due to the optical transition with an ultranarrow linewidth plays an essential role for our purpose. Furthermore, another striking property of AEAs is that the spin degrees of freedom which come purely from the nuclear spin $I$ offer $\mathrm{SU}(N=2 I+1)$ spins with $N \leq 10$ [29]. It is shown that the interplay between the optical coupling and the multicomponent spin structure of AEAs leads to various unique features in the laser-induced Kondo effect, in contrast to previous proposals of the Kondo effect using ultracold atoms [29-34].

Setup.-We consider ultracold fermionic AEAs in a three-dimensional optical lattice which consist of the ${ }^{1} S_{0}$ state and the ${ }^{3} P_{0}$ state. The fermionic annihilation operators of the ${ }^{1} S_{0}$ and ${ }^{3} P_{0}$ states at site $i$ are denoted as $c_{i \sigma}$ and $f_{i \sigma}$, respectively, and also as $c_{k \sigma}$ and $f_{k \sigma}$ for their Fourier transform. Here $\sigma=-I, \ldots, I$ labels the $z$ component of the nuclear spin. The system is initially prepared with the Hamiltonian

$$
\mathcal{H}_{0}=\sum_{\boldsymbol{k}, \sigma}\left[\varepsilon_{c}(\boldsymbol{k}) c_{\boldsymbol{k} \sigma}^{\dagger} c_{\boldsymbol{k} \sigma}+\varepsilon_{f}^{(0)} f_{\boldsymbol{k} \sigma}^{\dagger} f_{\boldsymbol{k} \sigma}\right]+U \sum_{i, \sigma<\sigma^{\prime}} n_{f i \sigma} n_{f i \sigma^{\prime}}
$$

with $n_{f i \sigma}=f_{i \sigma}^{\dagger} f_{i \sigma}$, where we denote the band dispersion of each orbital as $\varepsilon_{c}(\boldsymbol{k})$ and $\varepsilon_{f}^{(0)}$. The setup is illustrated in 
(a)

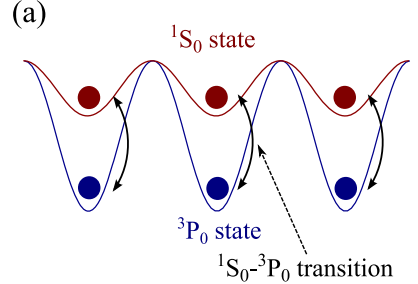

(c)

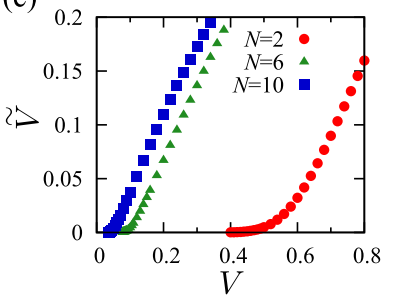

(b)

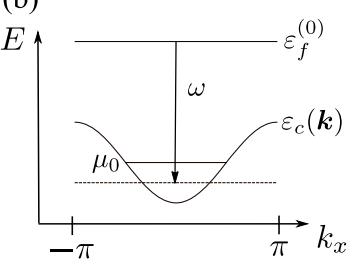

(d)

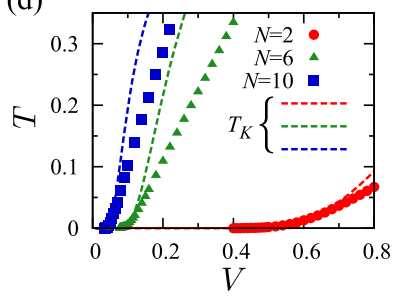

FIG. 1 (color online). (a) Schematic picture of the setup. Fermionic atoms in the ${ }^{1} S_{0}$ state can move between sites of the optical lattice, while those in the ${ }^{3} P_{0}$ state cannot. (b) The energy level shift after the gauge transformation. (c) Renormalized hybridization calculated with parameters $D=1, \varepsilon_{f}=-0.5$, $T_{0}=0, \mu_{0}=-0.1$, and $N=2,6,10$. (d) Effective temperatures calculated with the same parameters. The broken lines show the Kondo temperature $T_{K}$.

Fig. 1(a). Using the difference of polarizability of the two electronic states, an orbital-dependent optical lattice can be implemented in a manner such that the ${ }^{3} P_{0}$ state feels a deep lattice potential while the ${ }^{1} S_{0}$ state is confined weakly [29]. We assume that the ${ }^{3} P_{0}$ state is completely localized due to the strong confinement and thus is dispersionless, experiencing strong on-site repulsion, $U>0$. For the moment, we neglect other interatomic interactions. In the initial setup, we assume that the system is in thermal equilibrium with temperature $T_{0}$. The chemical potential for the ${ }^{1} S_{0}$ orbital is $\mu_{0}$, and the immobile ${ }^{3} P_{0}$ state is assumed to be singly occupied at each site.

At time $t=0$, we apply a monochromatic laser which induces the ${ }^{1} S_{0^{-}}{ }^{3} P_{0}$ transition allowed by the electric dipole coupling

$$
\mathcal{H}_{\text {mix }}=\theta(t) \sum_{i, \sigma, \sigma^{\prime}}\left(\boldsymbol{V} \cdot \boldsymbol{\sigma}_{\sigma \sigma^{\prime}} e^{i \boldsymbol{K} \cdot \boldsymbol{R}_{i}-i \omega t} f_{i \sigma}^{\dagger} c_{i \sigma^{\prime}}+\text { H.c. }\right) .
$$

Here we consider the simplest setup for the application of the laser, and $\theta(t)$ is the Heaviside step function. After $t=0$, the system evolves in time under the Hamiltonian, $\mathcal{H}_{0}+\mathcal{H}_{\text {mix }}$. The coupling coefficient in $\mathcal{H}_{\text {mix }}$ follows from matrix elements of $\boldsymbol{- d} \cdot \boldsymbol{E}$, where $\boldsymbol{d}$ is the electric dipole moment and $\boldsymbol{E}$ is the electric field of light. Using the Wigner-Eckart theorem [35], the matrix elements of $\boldsymbol{d}$ are proportional to those of the nuclear spin operator $\boldsymbol{\sigma}_{\sigma \sigma^{\prime}}$, since there is no electronic angular momentum.

Our central idea is that the Hamiltonian $\mathcal{H}=\mathcal{H}_{0}+\mathcal{H}_{\text {mix }}$ mimics the Anderson lattice model for heavy-fermion systems, and the hybridization term $\mathcal{H}_{\text {mix }}$ induces an

effective antiferromagnetic interaction between the orbitals [1,2]. After a gauge transformation $f_{i \sigma}(t)=$ $e^{i \boldsymbol{K} \cdot \boldsymbol{R}_{i}-i \omega t} f_{i \sigma}^{\prime}(t)$, the optical coupling takes a stepwise form in time. Hence, in this setup the hybridization term is suddenly turned on, and this corresponds to a "hybridization quench" problem of the Anderson lattice model. The transformation involves the energy level shift in the ${ }^{3} P_{0}$ orbital, $\varepsilon_{f} \equiv \varepsilon_{f}^{(0)}-\omega$, as depicted in Fig. 1(b), which comes from the time-derivative term in the Lagrangian.

Emergence of the Kondo effect under the laser field.-In this Letter, we focus on properties of the steady state under the irradiation. To analyze the Kondo effect arising from the Hamiltonian $\mathcal{H}$, we employ slave-boson mean-field theory [36-38], which becomes exact in the $N \rightarrow \infty$ limit ( $N=2 I+1$ is the number of spin components). As the first approximation, we take the strong-correlation limit $U \rightarrow \infty$ and neglect the double occupancy of the ${ }^{3} P_{0}$ state. Next, we split the $f$ operators into $f_{i \sigma}^{\prime}=b_{i}^{\dagger} \tilde{f}_{i \sigma}$ by introducing a slave-boson operator $b_{i}$, with a constraint $\sum_{\sigma} \tilde{f}_{i \sigma}^{\dagger} \tilde{f}_{i \sigma}+b_{i}^{\dagger} b_{i}=1$ at each site. To impose the constraint, the Lagrange multiplier field $\lambda_{i}$ is added to the Hamiltonian. After integrating out the fermions and taking a variation of the action with respect to $b_{i}$ and $\lambda_{i}$, we can obtain the saddle-point conditions in the (real-time) pathintegral formalism (the derivation is given in [39]). For simplicity, we set a homogeneous ansatz for the mean-field solution, neglecting the effect of the trap potential to atoms. We obtain the saddle-point conditions for the steady state as

$$
\begin{gathered}
\tilde{V}\left(\tilde{\varepsilon}_{f}-\varepsilon_{f}\right)+\frac{V^{2}}{2 N_{s}} \sum_{\boldsymbol{k}, \sigma, \sigma^{\prime}}\left(\hat{\boldsymbol{V}} \cdot \boldsymbol{\sigma}_{\sigma \sigma^{\prime}}\right)^{*} i G_{f c, \boldsymbol{k} \sigma \sigma^{\prime}}^{K}(t, t)=0, \\
\frac{1}{N_{s}} \sum_{\boldsymbol{k}, \sigma} i G_{f f, \boldsymbol{k} \sigma \sigma}^{<}(t, t)+\left(\frac{\tilde{V}}{V}\right)^{2}-1=0,
\end{gathered}
$$

where $G_{f f, \boldsymbol{k} \sigma \sigma}^{<}\left(t, t^{\prime}\right)=i\left\langle\tilde{f}_{\boldsymbol{k} \sigma}^{\dagger}(t) \tilde{f}_{\boldsymbol{k} \sigma}\left(t^{\prime}\right)\right\rangle$ and $G_{f c, \boldsymbol{k} \sigma \sigma^{\prime}}^{K}\left(t, t^{\prime}\right)=$ $-i\left\langle\left[\tilde{f}_{k \sigma}(t), c_{k \sigma^{\prime}}^{\dagger}\left(t^{\prime}\right)\right]\right\rangle$ are the lesser and Keldysh components of the Green's function, respectively [41]. $N_{s}$ denotes the number of sites. The mean fields $b$ and $\lambda$ (here we have dropped the site indices) are incorporated in the renormalized hybridization $\tilde{\boldsymbol{V}} \equiv \boldsymbol{V} b / \sqrt{N_{s}}$ and the renormalized energy level, $\tilde{\varepsilon}_{f} \equiv \varepsilon_{f}+\lambda / \sqrt{N_{s}}$. These renormalization effects are the manifestation of the strong correlation arising from the Kondo effect. We set $V=|\boldsymbol{V}|, \tilde{V}=|\tilde{\boldsymbol{V}}|$, and $\hat{\boldsymbol{V}}=\boldsymbol{V} /|\boldsymbol{V}|$.

After the gauge transformation, the Hamiltonian at $t>0$ is time independent. In this Letter, instead of solving the full time-dependent mean-field theory, we simply assume that the steady state after a long time is a thermal equilibrium state described by an effective temperature $T$ and a chemical potential $\mu$. Since the external driving leads to heating of the system, the temperature $T$ and the chemical potential $\mu$ after the application of the laser are 
different from $T_{0}$ and $\mu_{0}$. The Kondo effect occurs below the characteristic temperature, the so-called Kondo temperature $T_{K}$. If the effective temperature $T$ exceeds the Kondo temperature, the Kondo effect is washed out by the heating. To address the effect of heating by the application of the laser, we evaluate $T$ and $\mu$ using the energy conservation law and the particle number conservation law (the explicit forms are found in [39]), since the atoms are trapped in vacuum and well isolated from the environment. The parameters to be determined are hence $\left(\tilde{V}, \tilde{\varepsilon}_{f}, T, \mu\right)$, and they are obtained self-consistently from the four equations, namely, the two saddle-point conditions, Eqs. (3) and (4), and the two conservation laws.

The self-consistent equations are solved numerically. In the numerical calculation, we assume that the laser has $\pi$ polarization $\boldsymbol{V}=(0,0, V)$, and that the density of states of the ${ }^{1} S_{0}$ orbital is constant with finite bandwidth, $\rho(\varepsilon)=\rho_{0}=1 /(2 D),-D \leq \varepsilon \leq D$. The Kondo temperature $T_{K}$ is defined by the temperature where the slave boson starts to have a nonzero expectation value in thermal equilibrium [42], as $T_{K}=c_{N}(D-\mu)^{1 / N}(D+$ $\mu)^{1-1 / N} \exp \left[\left(\varepsilon_{f}-\mu\right) / A_{N} \rho_{0} V^{2}\right]$ where $A_{N}=N\left(N^{2}-1\right) / 12$ and $c_{N}$ is a weakly $N$-dependent constant of order one [39]. Figures 1(c) and 1(d) show the numerical solutions of the self-consistent equations starting from the zero-temperature initial state. The nonzero value of the renormalized hybridization, which entangles the localized ${ }^{3} P_{0}$ atoms with the ${ }^{1} S_{0}$ cloud, signals the emergence of the Kondo effect under the photoirradiation. The strongly renormalized value of $\tilde{V}$ leads to a hybridized band with a narrow Kondo gap [see Fig. 2(a)], and thus realizes a heavyfermion liquid. The effective temperature [Fig. 1(d)] is increased due to the heating effect caused by the irradiation. An important point is that the effective temperature is always lower than the Kondo temperature $T_{K}$, and thus the Kondo effect can be realized. If the initial states are at finite temperatures, we can obtain similar results, although in this case the Kondo effect appears above some finite laser strength [39]. If the Fermi temperature is of the order of $100 \mathrm{nK}$, the necessary laser strength (or Rabi frequency) is
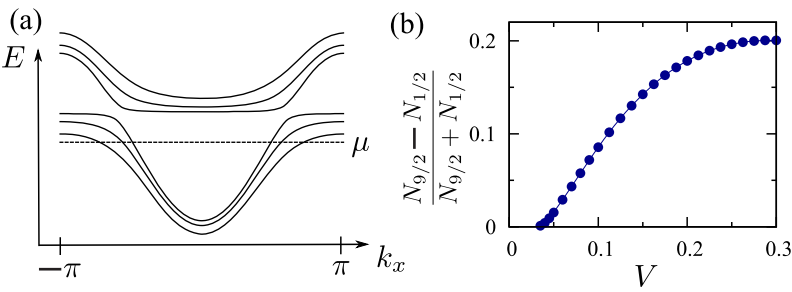

FIG. 2 (color online). (a) The renormalized quasiparticle band induced by the laser-induced Kondo effect for $N=6$. (b) Population imbalance induced by the Kondo effect. The $N=10$ case is shown, with parameters $D=1, \varepsilon_{f}=-0.8, T_{0}=0, \mu_{0}=-0.5$. $N_{\sigma}=n_{\sigma}+n_{-\sigma}$ is the particle number of each spin component. estimated to be a few $\mathrm{kHz}$, which is an achievable value in experiments.

Note that AEAs can offer a system with large- $N$ spin components, where $N=6$ for ${ }^{173} \mathrm{Yb}$ [20-25] and $N=10$ for ${ }^{87} \mathrm{Sr}$ [26-28]. The Kondo temperature, which is the underlying energy scale of the Kondo physics, rapidly increases with the number of components $N$. This means that the Kondo state is more stable in large- $N$ systems, and makes the observation of the Kondo effect more feasible in the large spin state with AEAs which is experimentally realized $[20-28,43]$.

We also comment on a role of the trap potential. Since the trap potential reduces $\varepsilon_{f}-\mu, T_{K}$ is lowest at the trap center, and there should appear a "mixed valence" region where $\varepsilon_{f} \lesssim \mu$ and the Kondo temperature is quite high. The existence of that region supports the feasibility to realize the Kondo liquid. However, we note that since the renormalization effect is weak in the mixed valence regime, a sharp Kondo effect may appear near the trap center.

Spin-selective renormalization.-The laser-induced Kondo state shows peculiar features owing to interplay between the optical coupling and the multicomponent spin structure of AEAs. In particular, the spin-dependent optical coupling leads to intriguing consequences with the Kondo state. The optical coupling for the $\pi$-polarized light becomes $\boldsymbol{V} \cdot \boldsymbol{\sigma}_{\sigma \sigma^{\prime}}=\sigma V \delta_{\sigma \sigma^{\prime}}$ and explicitly breaks the spin $\mathrm{SU}(N)$ invariance. Therefore, the laser-driven Kondo gaps are spin dependent, as seen in the quasiparticle band structure $\varepsilon_{\sigma \pm}(\boldsymbol{k})=\frac{1}{2}\left\{\varepsilon_{c}(\boldsymbol{k})+\tilde{\varepsilon}_{f} \pm \sqrt{\left[\varepsilon_{c}(\boldsymbol{k})-\tilde{\varepsilon}_{f}\right]^{2}+4|\sigma \tilde{V}|^{2}}\right\}$ [illustrated in Fig. 2(a)] which is obtained by diagonalizing the mean-field Hamiltonian. This gives rise to spin-dependent effective masses of quasiparticles, since the quasiparticle band is more flattened in the higher spin components at the Fermi energy. As a result, the laser-induced Kondo effect selectively renormalizes the effective masses of higher spin components, beside light quasiparticles of lower spin components. These consequences are in marked contrast to the $\mathrm{SU}(N)$-symmetric Kondo effect proposed for ultracold atoms previously [29-34], where the all spin components are completely degenerate.

These characteristic spin-dependent structures are reflected in physical observables. The spin-dependent quasiparticle band yields population imbalance between the spin components. In Fig. 2(b), we have plotted the population imbalance. The population imbalance, which can be directly measured by cold-atom experiments, inherits both the spin-dependent nature and nonperturbative $V$ dependence in the laser-induced Kondo effect. The Kondo states can also be measured by density profiles of the atomic cloud. For $N=2$, and when the ${ }^{1} S_{0}$ orbital is half-filled in the lattice, the lower hybridized band formed by the Kondo effect is completely filled: this phase is called the Kondo insulator. Reflecting the formation of the Kondo insulator, a density plateau of the atomic cloud appears in some region of the atomic cloud [31,32]. Similarly, in the 
case of $N>2$, reflecting the spin-dependent Kondo gaps, a half-metallic phase composed of a completely filled band (the Kondo insulator) and partially filled Kondo metals can be formed. Correspondingly, the density plateau may also be spin dependent for $N>2$.

Effect of exchange interactions: Tuning anisotropy.-So far we have described the laser-induced Kondo effect using the minimal Hamiltonian $\mathcal{H}_{0}+\mathcal{H}_{\text {mix }}$, which includes only interactions between the ${ }^{3} P_{0}$ states. In reality, other interactions also exist [29]. Among them, strong interorbital $\mathrm{SU}(N)$ exchange interactions observed in recent experiments $[24,25,28]$ play the most crucial role for the Kondo physics. Here we consider how these interactions affect the laser-induced Kondo effect. The $\mathrm{SU}(N)$ exchange interaction is described by

$$
\mathcal{H}_{\mathrm{ex}}=V_{\mathrm{ex}} \sum_{i, \sigma, \sigma^{\prime}} c_{i \sigma}^{\dagger} f_{i \sigma^{\prime}}^{\dagger} c_{i \sigma^{\prime}} f_{i \sigma},
$$

where $V_{\mathrm{ex}}>0\left(V_{\mathrm{ex}}<0\right)$ is the ferromagnetic (antiferromagnetic) case. To gain insight into the Kondo effect in the case where both the exchange interaction and photoinduced hybridization exist, it is convenient to consider situations in which the laser frequency is highly off resonant and optical transitions are well suppressed. In this case, we can derive an effective Hamiltonian in the Hilbert subspace restricted to $\sum_{\sigma} n_{f i \sigma}=1$, using a standard treatment by the Schrieffer-Wolff transformation [44]. At the second order of $V$, we obtain an effective Kondo exchange interaction by the $\pi$-polarized laser as

$$
\mathcal{H}_{\mathrm{eff}}=\sum_{i, \sigma, \sigma^{\prime}}\left(V_{\mathrm{ex}}-J \sigma \sigma^{\prime}\right) c_{i \sigma}^{\dagger} f_{i \sigma^{\prime}}^{\dagger} c_{i \sigma^{\prime}} f_{i \sigma},
$$

where $J \simeq 2 V^{2} /\left|\varepsilon_{f}\right|>0$ [45]. The resulting exchange interaction, Eq. (6), is anisotropic, and we find that the "tuning" of the anisotropy by the laser leads to an intriguing consequence. The effect of the anisotropic exchange coupling can be deduced from the renormalization group (RG) flow using Anderson's poor-man's scaling for the case of Kondo impurity, particularly in the case of $N=2$ [46]. The coupling constants $J_{\perp}=V_{\text {ex }}+J / 4$ and $J_{\|}=$ $V_{\text {ex }}-J / 4$ change along a trajectory on the RG flow diagram as depicted in Fig. 3 when we increase $J$. When we turn on the laser in the case of $V_{\mathrm{ex}}>0$, the coupling constants immediately change from an irrelevant coupling into a relevant one; namely, the laser-induced Kondo effect occurs, despite the bare ferromagnetic exchange coupling. This fact is in sharp contrast to the proposals in Refs. [29,31,32]. The behavior of the RG flow from the weak-coupling fixed point to the strong-coupling one may be observed as a crossover in the temperature dependence of the laser-induced Kondo effect.

In the case of $V_{\mathrm{ex}}<0$, where the bare exchange coupling is relevant, the Kondo effect owing to $V_{\text {ex }}$ (as described in

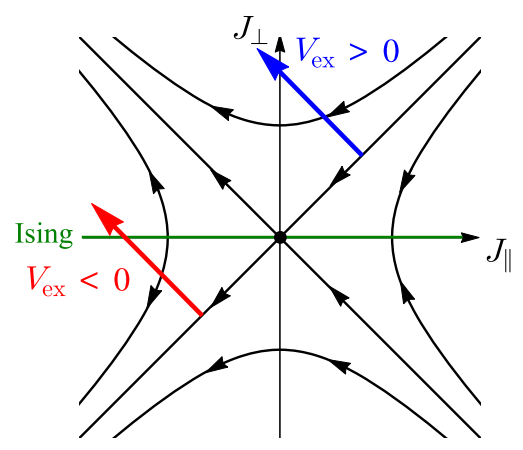

FIG. 3 (color online). The renormalization group flow for the case of the Kondo impurity for $N=2$. The blue (red) line shows a trajectory of the coupling constants when we apply the external laser in the case of $V_{\mathrm{ex}}>0\left(V_{\mathrm{ex}}<0\right)$.

Refs. [31,32]) takes place. This Kondo effect is suppressed by the laser application. At $J=4\left|V_{\text {ex }}\right|$, the Kondo effect vanishes since the interaction is purely Ising-type, $J_{\perp}=0$. After that, as seen in the flow diagram, the Kondo effect revives and the system is governed by another strongcoupling fixed point which is described in this Letter. In other words, the laser gives a novel method to induce a reentrant Kondo effect across the Ising point.

For $N>2$, the situation is more complicated since more coupling constants appear in the Hamiltonian. We note that there is no "Ising" point where all spin-flip terms simultaneously vanish in this case, and thus the reentrant behavior of the Kondo effect may be most prominent in the case of $N=2$. A full analysis needs further investigations and is left for future work.

Summary.-We have proposed a possible realization of the laser-induced Kondo state, using ultracold alkalineearth atoms in an optical lattice. It has been shown that the emergent Kondo effect under the application of the laser field overwhelms the heating effect, thereby realizing the orbital-spin entangled singlets and the heavy-fermion liquid. Furthermore, we have elucidated peculiar Kondo physics arising from the specific form of optical coupling, such as the spin-selective renormalization of effective masses and the nontrivial competition between the bare exchange coupling and the laser-induced hybridization which leads to a novel crossover or reentrant behavior of the Kondo effect.

The laser-induced Kondo effect proposed here provides a new avenue to study real-time dynamics of strongly correlated systems in nonequilibrium situations. One of interesting future perspectives is to address the transient process, where the Kondo singlet develops towards the steady state described in this Letter. Another interesting issue may be related to magnetic properties. Here we have only considered paramagnetic states, but Ruderman-KittelKasuya-Yosida interactions mediated by the optical transitions are expected to cause various magnetic orders competing with the Kondo effect. This may lead to novel 
quantum criticality driven by external laser fields. These issues are expected to be clarified in future studies.

We are thankful to Yoshiro Takahashi for valuable discussions. This work was supported by KAKENHI (Grants No. 25400366, No. 14J01328, and No. 15H05855). M. N. thanks JSPS for the support from a Research Fellowship for Young Scientists.

[1] A. C. Hewson, The Kondo Problem to Heavy Fermions (Cambridge University Press, Cambridge, 1993).

[2] P. Coleman, in Handbook of Magnetism and Advanced Magnetic Materials, edited by H. Kronmueller and S. Parkin (John Wiley \& Sons, New York, 2007).

[3] D. Jaksch and Z. Zoller, Ann. Phys. (Amsterdam) 315, 52 (2005).

[4] J. Dalibard, F. Gerbier, G. Juzeliūnas, and P. Öhberg, Rev. Mod. Phys. 83, 1523 (2011).

[5] N. H. Lindner, G. Refael, and V. Galitski, Nat. Phys. 7, 490 (2011).

[6] M. Eckstein and P. Werner, Phys. Rev. Lett. 110, 126401 (2013).

[7] S. Takayoshi, M. Sato, and T. Oka, Phys. Rev. B 90, 214413 (2014).

[8] N. R. Cooper, Phys. Rev. Lett. 106, 175301 (2011).

[9] L. Jiang, T. Kitagawa, J. Alicea, A. R. Akhmerov, D. Pekker, G. Refael, J. I. Cirac, E. Demler, M. D. Lukin, and P. Zoller, Phys. Rev. Lett. 106, 220402 (2011).

[10] M. Nakagawa and N. Kawakami, Phys. Rev. A 89, 013627 (2014).

[11] H. Aoki, N. Tsuji, M. Eckstein, M. Kollar, T. Oka, and P. Werner, Rev. Mod. Phys. 86, 779 (2014).

[12] M. Heyl and S. Kehrein, Phys. Rev. B 81, 144301 (2010).

[13] P. Werner and M. Eckstein, Phys. Rev. B 86, 045119 (2012).

[14] M. H. Hettler and H. Schoeller, Phys. Rev. Lett. 74, 4907 (1995).

[15] P. Nordlander, M. Pustilnik, Y. Meir, N. S. Wingreen, and D. C. Langreth, Phys. Rev. Lett. 83, 808 (1999).

[16] T. V. Shahbazyan, I. E. Perakis, and M. E. Raikh, Phys. Rev. Lett. 84, 5896 (2000).

[17] C. Latta, F. Haupt, A. Weichselbaum, M. Claassen, W. Wuester, P. Fallahi, S. Faelt, L. Glazman, J. von Delft, H. E. Türeci, and A. Imamoglu, Nature (London) 474, 627 (2011).

[18] H. E. Türeci, M. Hanl, M. Claassen, A. Weichselbaum, T. Hecht, B. Braunecker, A. Govorov, L. Glazman, A. Imamoglu, and J. von Delft, Phys. Rev. Lett. 106, 107402 (2011).

[19] B. Sbierski, M. Hanl, A. Weichselbaum, H. E. Türeci, M. Goldstein, L. I. Glazman, J. von Delft, and A. İmamoğlu, Phys. Rev. Lett. 111, 157402 (2013).

[20] T. Fukuhara, Y. Takasu, M. Kumakura, and Y. Takahashi, Phys. Rev. Lett. 98, 030401 (2007).

[21] S. Taie, Y. Takasu, S. Sugawa, R. Yamazaki, T. Tsujimoto, R. Murakami, and Y. Takahashi, Phys. Rev. Lett. 105, 190401 (2010).

[22] S. Taie, R. Yamazaki, S. Sugawa, and Y. Takahashi, Nat. Phys. 8, 825 (2012).
[23] G. Pagano, M. Mancini, G. Cappellini, P. Lombardi, F. Schäfer, H. Hu, X.-J. Liu, J. Catani, C. Sias, M. Inguscio, and L. Fallani, Nat. Phys. 10, 198 (2014).

[24] G. Cappellini, M. Mancini, G. Pagano, P. Lombardi, L. Livi, M. Siciliani de Cumis, P. Cancio, M. Pizzocaro, D. Calonico, F. Levi, C. Sias, J. Catani, M. Inguscio, and L. Fallani, Phys. Rev. Lett. 113, 120402 (2014).

[25] F. Scazza, C. Hofrichter, M. Höfer, P. C. D. Groot, I. Bloch, and S. Fölling, Nat. Phys. 10, 779 (2014).

[26] B. J. DeSalvo, M. Yan, P. G. Mickelson, Y. N. Martinez de Escobar, and T. C. Killian, Phys. Rev. Lett. 105, 030402 (2010).

[27] M. J. Martin, M. Bishof, M. D. Swallows, X. Z. C. Benko, J. von Stecher, A. V. Gorshkov, A. M. Rey, and J. Ye, Science 341, 632 (2013).

[28] X. Zhang, M. Bishof, S. L. Bromley, C. V. Kraus, M. S. Safronova, P. Zoller, A. M. Rey, and J. Ye, Science 345, 1467 (2014).

[29] A. V. Gorshkov, M. Hermele, V. Gurarie, C. Xu, P. S. Julienne, J. Ye, P. Zoller, E. Demler, M. D. Lukin, and A. M. Rey, Nat. Phys. 6, 289 (2010).

[30] B. Paredes, C. Tejedor, and J. I. Cirac, Phys. Rev. A 71, 063608 (2005).

[31] M. Foss-Feig, M. Hermele, and A. M. Rey, Phys. Rev. A 81, 051603 (2010).

[32] M. Foss-Feig, M. Hermele, V. Gurarie, and A. M. Rey, Phys. Rev. A 82, 053624 (2010).

[33] J. Bauer, C. Salomon, and E. Demler, Phys. Rev. Lett. 111, 215304 (2013).

[34] Y. Nishida, Phys. Rev. Lett. 111, 135301 (2013).

[35] L. D. Landau and E. M. Lifshitz, Quantum Mechanics: Non-Relativistic Theory, 3rd ed. (Butterworth-Heinemann, Oxford, 1981).

[36] D. M. Newns and N. Read, Adv. Phys. 36, 799 (1987).

[37] P. Coleman, Phys. Rev. B 35, 5072 (1987).

[38] A. J. Millis and P. A. Lee, Phys. Rev. B 35, 3394 (1987).

[39] See Supplemental Material at http://link.aps.org/ supplemental/10.1103/PhysRevLett.115.165303, which includes Ref. [40], for the derivation of the mean-field equations, the derivation of the Kondo temperature, and numerical calculations at finite initial temperatures.

[40] Z. Ratiani and A. Mitra, Phys. Rev. B 79, 245111 (2009).

[41] A. Kamenev, Field Theory of Non-Equilibrium Systems (Cambridge University Press, Cambridge, 2011).

[42] S. Burdin, A. Georges, and D. R. Grempel, Phys. Rev. Lett. 85, 1048 (2000).

[43] For $N=2$, fluctuation effects beyond the mean-field theory are significant. It is known that the fluctuation effects reduce $T_{K}$ from the mean-field result. However, since the fluctuation also affects the energy spectrum, the effective temperature beyond the mean-field result is an interesting issue for a future study.

[44] J. R. Schrieffer and P. A. Wolff, Phys. Rev. 149, 491 (1966).

[45] A potential scattering term from the Schrieffer-Wolff transformation can be absorbed into the chemical potential. We also remark that this transformation is not valid near the edge of the atomic cloud.

[46] P. W. Anderson, J. Phys. C 3, 2436 (1970). 